7 Shiohara T, Mizukawa Y: Fixed drug eruption: easily overlooked but needing new respect. Dermatology 2002;205:103-104.

8 Bernand S, Scheidegger EP, Dummer R, Burg G: Multifocal fixed drug eruption to paracetamol, tropisetron and ondansetron induced by interleukin 2. Dermatology 2000;201:148-150.

Risa Tamagawa-Mineoka

Department of Dermatology, Kyoto Prefectural University of Medicine Graduate School of Medical Science

465, Kajii-cho, Kawaramachi-Hirokoji, Kamigyo-ku

Kyoto 602-8566 (Japan)

Tel./Fax +81 75251 5586, E-Mail risat@koto.kpu-m.ac.jp

Dermatology 2008;216:183

DOI: $10.1159 / 000111521$

\section{Symmetrical Drug-Related Intertriginous and Flexural Exanthema}

\section{Ronni Wolf, Batya Davidovici}

Dermatology Unit, Kaplan Medical Center, Rechovot, Israel

Key Words

Symmetrical drug-related intertriginous and flexural exanthema $\cdot$ Radio contrast medium $\cdot$ Drug rash with eosinophilia and systemic symptoms

We congratulate Arnold et al. [1] for their interesting report on symmetrical drug-related intertriginous and flexural exanthema (SDRIFE, baboon syndrome). We especially commend the first criterion of this syndrome stating, what we emphasized some 15 years ago [2], that SDRIFE should be distinguished from hematogenous or systemic contact-type dermatitis, as suggested by Andersen et al. [3], when they first reported this syndrome.

We would like to make two comments that by no means detract from the scientific and educational value of this paper.

First, the authors state that 'the particular pattern of SDRIFE has not been observed so far' as a reaction to radio contrast medium (RCM). We refer them to our report [4] describing 11 patients with SDRIFE, one of them (case 10) due to RCM, thus supporting a causal relationship between RCM and SDRIFE.

Second, the fifth criterion of SDRIFE is absence of systemic symptoms and signs, which according to the authors [1] is important to distinguish SDRIFE from drug rash with eosinophilia and systemic symptoms (DRESS). Here, we disagree. We contend that a patient with an eruption that has the cutaneous appearance of SDRIFE but with additional systemic organ involvement should still be classified as SDRIFE and not as DRESS. As we had suggested in an earlier publication [5], we believe that all drug eruptions - involvement of other organ systems notwithstanding - are first and foremost dermatological diseases and, as such, they should be classified according to the cutaneous lesions. In other words, SDRIFE with systemic symptoms and/or eosinophilia should be defined as SDRIFE with systemic organ involvement and not as DRESS.

\section{References}

1 Arnold AW, Hausermann P, Bach S, Bircher AJ: Recurrent flexural exanthema (SDRIFE or baboon syndrome) after administration of two different iodinated radio contrast media. Dermatology 2007;214:8993.

2 Wolf R, Elman M, Brenner S: Drug-induced 'intertrigo'. Int J Dermatol 1993;32:515-516.

3 Andersen KE, Hjorth N, Menne T: The baboon syndrome: systemically induced allergic contact dermatitis. Contact Dermatitis 1984;10: 97-100.

4 Wolf R, Orion E, Matz H: The baboon syndrome or intertriginous drug eruption: a report of eleven cases and a second look at its pathomechanism. Dermatol Online J 2003;9:2.

5 Wolf R, Matz H, Marcos B, Orion E: Drug rash with eosinophilia and systemic symptoms vs toxic epidermal necrolysis: the dilemma of classification. Clin Dermatol 2005;23:311-314.

Ronni Wolf, MD

Dermatology Unit, Kaplan Medical Center

IL-76100 Rechovot (Israel)

Fax +97299560978

E-Mailwolf_r@netvision.net.il
Dermatology 2008;216:183-184
DOI: $10.1159 / 000111522$

\section{Symmetrical Drug-Related Intertriginous and Flexural Exanthema - Reply}

Andreas J. Bircher, Andreas Arnold, Silvy Bach, Peter Häusermann Allergy Unit, Department of Dermatology, University Hospital, Basel, Switzerland

\section{Key Words}

Symmetrical drug-related intertriginous and flexural exanthema Flexural exanthema $\cdot$ Barium sulfate

We thank Dr. Wolf and Dr. Davidovici for their thoughtful comments on our case report [1]

We regret that the clinical case observation of a 79-year-old woman who developed the particular pattern of flexural exanthema a few hours after receiving barium sulfate by the gastrointestinal route [2] was not mentioned in our article. Since barium sulfate contains some additives, another allergen could have been responsible. To our best knowledge, iodinated radio contrast media have not been implicated in symmetrical drug-related intertriginous and flexural exanthema (SDRIFE) so far.

We definitely agree with the authors that the description and diagnosis of cutaneous drug eruptions often require the expertise of a dermatologist. However, most patients with cutaneous drug eruptions are initially seen by nondermatologists such as general practitioners. Particularly these physicians should be aware of the specific danger signs of the severer drug eruptions with internal organ involvement [3]. On the other hand, we believe that it is less a particular morphological pattern, such as a maculopapular exanthema or SDRIFE, than the danger signs of severer reactions, 
such as high fever, general malaise, eosinophilia or hepatic involvement, which should alert the treating physicians to suspect a severe drug reaction and to seek advice of a specialist.

Neither in the literature nor in our many personal observations of patients with SDRIFE have we been able to identify cases, who also suffered from severe systemic symptoms or signs, such as high fever, cytopenia, eosinophilia or hepatitis. Also e.g. drug rash with eosinophilia and systemic symptoms is typically associated with maculopapular lesions, which are an integral part of the complete syndrome, and toxic epidermal necrolysis often begins with a primarily innocuous macular sometimes painful exanthema [4]. This implies that independently of the type of cutaneous manifestation, the presence of alert signs should always be searched for and respected.

If in the future cases with the particular morphological pattern of SDRIFE and severe systemic signs should be published, a reevaluation of our currently proposed criteria might be necessary. Currently, however, we hypothesize that SDRIFE belongs to the clinically heterogenous group of T-cell-mediated macular, maculopapular, papular and pustular exanthemas which may be associated with mild systemic symptoms, such as mild fever or pruritus, but it is not associated with an involvement of internal organs. The reason for the particular distribution of the exanthema remains to be elucidated.

\section{References}

1 Arnold AW, Häusermann P, et al: Recurrent flexural exanthema (SDRIFE or baboon syndrome) after administration of two different iodinated radio contrast media. Dermatology 2007;214:89-93.

2 Wolf R, Orion E, et al: The baboon syndrome or intertriginous drug eruption: a report of eleven cases and a second look at its pathomechanism. Dermatol Online J 2003;9:2.

3 Bircher AJ: Symptoms and danger signs in acute drug hypersensitivity. Toxicology 2005;209:201-207.

4 Häusermann P, Harr T, et al: Baboon syndrome resulting from systemic drugs: is there strife between SDRIFE and allergic contact dermatitis syndrome? Contact Dermatitis 2004;51:297-310.

Prof. Dr. Andreas J. Bircher, MD

Allergy Unit, Department of Dermatology, University Hospital Basel Petersgraben 4, CH-4031 Basel (Switzerland) Tel. +41 6126543 59, Fax +41 612654885

E-Mail andreas.bircher@unibas.ch 